\title{
Data-Driven Superheating Control of Organic Rankine Cycle Processes
}

\author{
Jianhua Zhang $\mathbb{D},{ }^{1}$ Xiao Tian, ${ }^{2}$ Zhengmao $\mathrm{Zhu}^{2}$ and Mifeng Ren $^{3}$ \\ ${ }^{1}$ State Key Laboratory of Alternate Electrical Power System with Renewable Energy Sources, North China Electric Power University, \\ Beijing 102206, China \\ ${ }^{2}$ School of Control and Computer Engineering, North China Electric Power University, Beijing 102206, China \\ ${ }^{3}$ College of Electrical and Power Engineering, Taiyuan University of Technology, Taiyuan 030024, China
}

Correspondence should be addressed to Jianhua Zhang; zjhncepu@163.com

Received 13 July 2018; Accepted 9 September 2018; Published 21 October 2018

Guest Editor: Jing $\mathrm{Na}$

Copyright (C) 2018 Jianhua Zhang et al. This is an open access article distributed under the Creative Commons Attribution License, which permits unrestricted use, distribution, and reproduction in any medium, provided the original work is properly cited.

In this paper, a data-driven superheating control strategy is developed for organic Rankine cycle (ORC) processes. Due to nonGaussian stochastic disturbances imposed on heat sources, the quantized minimum error entropy (QMEE) is adopted to construct the performance index of superheating control systems. Furthermore, particle swarm optimization (PSO) algorithm is applied to obtain optimal control law by minimizing the performance index. The implementation procedures of the presented superheating control system in an ORC-based waste heat recovery process are presented. The simulation results testify the effectiveness of the presented control algorithm.

\section{Introduction}

Organic Rankine cycle (ORC) processes have been widely used to utilize low-grade thermal energy [1-4]. The energy efficiency of an ORC system is closely related to the thermodynamic states of working fluid at various components in the cycle. The superheating is one of the key operating parameters involved with safety and energy efficiency; hence, superheating control plays an important role in organic Rankine cycle (ORC) processes. However, it is not easy to design a high-quality superheating control system for ORC processes because ORC processes are complex in terms of nonlinearities, coupling, and stochastic disturbances.

Some efforts have been made to develop superheating control algorithms for ORC processes [5-15]. In [6], traditional PID controller was applied to control the superheating of an ORC-based waste heat recovery process by manipulating pump flow rate. Combining PID controller with feedforward controller, a composite controller was developed for superheating control systems [7-9]. Another compound controller was proposed for designing ORC control system by incorporating a linear quadratic regulator with a PI controller [10]. A constrained model predictive controller was applied into a controlled ORC system to deal with constraints on inputs and outputs [11]. Later on, auto disturbances rejection, gain scheduling, and robust control strategies were applied to ORC processes in [13-15], respectively. In practical ORC processes with stochastic disturbances, the abovementioned control methods may be a little bit strict or conservative. In [12], generalized minimum variance controller was employed to deal with stochastic disturbances induced from heat sources, in which the key goal is to minimize the uncertainties of the closed-loop stochastic systems. However, stochastic disturbances that existed in ORC processes are not necessarily Gaussian; the spread area of the tracking error cannot be indicated precisely using variance or mean value.

With the rapid development of precision instrument, communication network, statistical analysis, image processing, and data processing technology, the probability density function (PDF) can be measured directly in many actual industrial processes. Recently, a series of control strategies for the output PDF have been developed [16-18]. On the other hand, the tracking error has played an important role 
for assessing closed-loop control performance; some control strategies have also been proposed for non-Gaussian systems based on minimizing entropy of tracking error [19-23]. In addition, entropy optimization principle has been utilized to design filter [24] and machine learning as well [25].

The most summarized argument of entropy is $(h, \varphi)$ -entropy, which has been employed to form performance index for control systems [26, 27]. Renyi entropy [28] is the most significant and commonly applied. In order to decrease the computational complexity of the entropy, a quantized minimum error entropy (QMEE) criterion was proposed in [29].

In this paper, following the recent developments on shape control of the output PDF, tracking control, and information theoretic learning using minimum error entropy principle, we cast superheating control of ORC processes into a stochastic control framework. Within this framework, a data-driven tracking control strategy is further investigated for ORC systems with non-Gaussian disturbances.

The remaining of this paper is organized as follows: Section 2 describes the ORC-based waste heat recovery process. Section 3 presents the proposed superheating controller using a quantized minimum error entropy criterion and particle swarm optimization (PSO) technique. The simulation results are then shown in Section 4. Finally, several conclusions are given in Section 5.

\section{ORC Process}

An ORC-based waste heat recovery power plant shown in Figure 1 converts waste heat into electrical power. The organic working fluid in the evaporator is heated up into a superheated vapour state. The heated vapour enters the turbine expander and generates power energy. The working fluid after expansion is then cooled to liquid state in the condenser.

In order to ensure the safety and energy conversion efficiency of the ORC process, the temperature of working fluid at the outlet of evaporator must be controlled within a proper range; hence, superheating that is one of the most important parameters in ORC processes is usually controlled by manipulating the rotating speed of the pump.

The physical model of the evaporator was established based on both mass balance and energy balance equations in our previous work $[10,11]$, where both sides of working fluid and waste heat were analyzed based on some proper assumptions and necessary simplification. The model of the pump was also built based on similarity principle in [10, 11]. The quality of waste heat characterized by the inlet temperature and the mass flow rate of waste heat has influence on the superheating. In practical ORC processes, the disturbances on the temperature and the mass flow rate of waste heat are not necessarily Gaussian. Therefore, the dynamics of an ORC process is nonlinear and non-Gaussian; the discretized model of controlled ORC superheating processes can be formulated as follows:

$$
y_{k}=f\left(y_{k-1}, \ldots, y_{k-n}, u_{k}, u_{k-1}, \ldots, u_{k-m}, w_{k}, v_{k}\right) \text {, }
$$

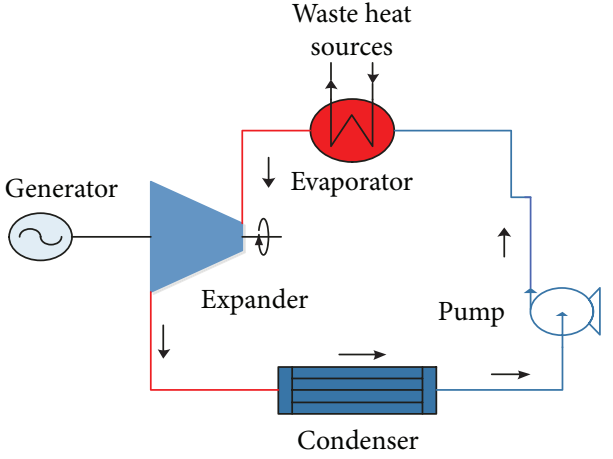

FIGURE 1: Schematic diagram of ORC-based waste heat recovery.

where $f(\cdot)$ is a known nonlinear function that represents the ORC superheating process dynamics. $y_{k}$ and $u_{k}$ are the superheating and the rotating speed of the pump, respectively. $w_{k}$ and $v_{k}$ are the mass flow rate and the temperature of waste heat source, respectively. In practice, the stochastic disturbances on the mass flow rate and the temperature of waste heat source are usually bounded, generally non-Gaussian, and mutually independent. Hence, the superheating $y_{k}$ and the tracking error $e_{k}$ are also non-Gaussian stochastic variables. Denote the set point of the superheating as $r_{k}$, the tracking error $e_{k}$ can be described by

$$
\begin{aligned}
e_{k} & =r_{k}-y_{k} \\
& =r_{k}-f\left(y_{k-1}, \ldots, y_{k-n}, u_{k}, u_{k-1}, \ldots, u_{k-m}, w_{k}, v_{k}\right) \\
& =g\left(y_{k-1}, \ldots, y_{k-n}, u_{k}, u_{k-1}, \ldots, u_{k-m}, w_{k}, v_{k}, r_{k}\right) \\
& =g\left(\eta_{k}, u_{k}, w_{k}, v_{k}\right),
\end{aligned}
$$

where $g(\cdot)$ is a known nonlinear function; $\eta_{k}=\left(y_{k-1}\right.$, $\left.\ldots, y_{k-n}, u_{k-1}, \ldots, u_{k-m}, r_{k}\right)^{T}$ is a known term at instant $k$. The PDF of the tracking error $e_{k}$ can then be formulated using probability theory when the PDFs of both the mass flow rate and the temperature of waste heat source are known. In practical ORC processes, the PDFs of the tracking error, the mass flow rate, and the temperature of waste heat source can be directly obtained by numerical estimation methods. Likewise, the entropy can be estimated; for example, the information potential that is the argument of the logarithm in Renyi entropy can be computed by a double summation over all samples. In this context, datadriven superheating control strategy can be investigated in this work.

Remark 1. When the PDFs of the mass flow rate and the temperature of waste heat source are known, the evolution of the PDF of the tracking error can be obtained based on (2) using probability theory; the relationship between the PDF of the tracking error and control input can be revealed. In addition, (2) can be used to develop model-based control algorithms or analyze stability of closed-loop control systems. 


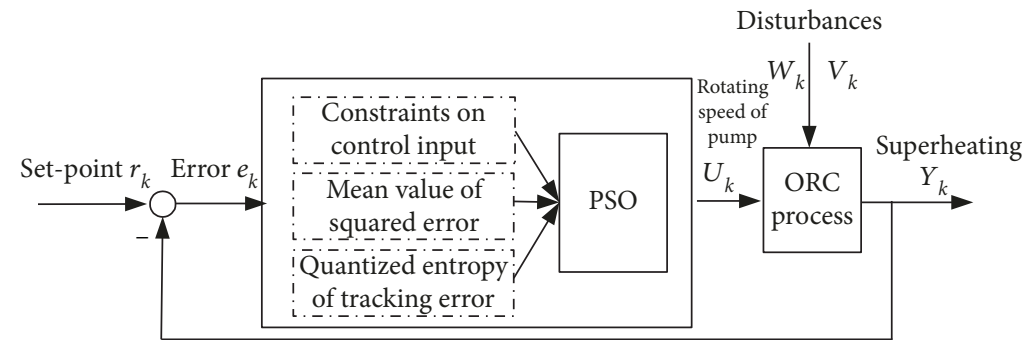

FIGURE 2: Schematic diagram of the proposed control system.

\section{Superheating Control of ORC Process}

3.1. Schematic Diagram. It has been demonstrated by experimental studies and theoretical analysis that superheating plays an important role in the safety and efficiency of ORC processes. A data-driven stochastic control method is proposed to ensure the superheating $y_{k}$ approach to its set point $r_{k}$. Figure 2 shows the control diagram, in which the controlled variable and the manipulated variable are the superheating and the rotating speed of the pump, respectively. The fluctuations of superheating can be represented by the shape of its PDF. As a result, the entropy of the tracking error can be used to represent the uncertainty of superheating or its tracking error.

3.2. Performance Index. It is clear from (2) that the PDF of the tracking error is controlled by manipulating the rotating speed of the pump. Ideally, the goal of designing the superheating controller is that the shape of the PDF of the tracking error becomes as narrow as possible. It means that the entropy of the tracking error $H\left(e_{k}\right)$ should be minimized. In addition, the mean value of the squared tracking error $E\left(e_{k}^{2}\right)=\int\left(\tau^{2} \rho_{e_{k}}\left(\eta_{k}, u_{k}, \tau\right)\right) d \tau$ should also be included to drive the tracking error approach to zero. Moreover, the control energy should be minimized as well. In this context, the following performance index is employed:

$$
J\left(u_{k}\right)=R_{1} H\left(e_{k}\right)+R_{2} E\left(e_{k}^{2}\right)+\frac{1}{2} R_{3} u_{k}^{2},
$$

where $R_{1}$ and $R_{2}$ are weights assigned for the entropy of the tracking error and the mean value of the squared tracking error function, respectively, and $R_{3}$ is the weighting factor for the control input. Consequently, the manipulated variable can be solved by minimizing the performance index at instant $k$.

The entropy of the tracking error $H\left(e_{k}\right)$ can be estimated via estimating the information potential. Although it can be realized with an affordable computational burden, it is necessary to estimate the entropy as soon as possible for large-scale datasets so that the real-time ability of the control system can be improved.

The quadratic information potential $I P_{2}\left(e_{k}\right)=\int \gamma_{e_{k}}^{2}\left(\eta_{k}\right.$, $\left.u_{k}, \tau\right) d \tau$ is an alternative way to characterize the quadratic entropy of tracking error $\mathrm{H}_{2}\left(e_{k}\right)$. Further on, the quantized quadratic information potential proposed in [29] can be applied to deal with computational complexity of the information potential by simplifying the inner summation. Therefore, the performance index (3) can be reformulated by

$$
J\left(u_{k}\right)=R_{1} \frac{1}{I P_{2}^{Q}\left(e_{k}\right)}+R_{2} E\left(e_{k}^{2}\right)+\frac{1}{2} R_{3} u_{k}^{2}
$$

where $I P_{2}^{Q}\left(e_{k}\right)$ is the quantized quadratic information potential, which can be estimated as follows:

$$
\begin{aligned}
I P_{2}^{Q}\left(e_{k}\right) & =\frac{1}{N^{2}} \sum_{i=k-N+1}^{k} \sum_{j=k-N+1}^{k} G_{\sqrt{2} \sigma}\left(e_{i}-e_{j}\right) \\
& \approx \frac{1}{N^{2}} \sum_{i=k-N+1}^{k} \sum_{j=k-N+1}^{k} G_{\sqrt{2} \sigma}\left(e_{i}-Q\left[e_{j}\right]\right) \\
& =\frac{1}{N^{2}} \sum_{i=k-N+1}^{k}\left(\sum_{m=k-N+1}^{M} M_{m} G_{\sqrt{2} \sigma}\left(e_{i}-c_{m}\right)\right) \\
& =\frac{1}{N} \sum_{i=k-N+1}^{k} \frac{\sum_{m=k-N+1}^{M} M_{m} G_{\sqrt{2} \sigma}\left(e_{i}-c_{m}\right)}{N},
\end{aligned}
$$

where $N$ is the number of error samples. $Q[\cdot]$ stands for a quantizer with a codebook containing $M$ real valued code words, which can map the error samples into the $M$ code words in the codebook. $G_{\sigma}(x)=(1 / \sqrt{2 \pi \sigma}) \exp \left(-\left(x^{2} / 2 \sigma^{2}\right)\right)$ is the Gaussian kernel with bandwidth $\sigma$.

It can be observed that the quantizer plays a significant role. A competent and simple quantizer was proposed in [30]. Following [31], the detailed procedures to estimate the quantized quadratic information potential of the tracking error using $N$ error samples within a sliding window at instant $k$ are summarized as Algorithm 1.

In addition, the mean value of the squared tracking error $E\left(e_{k}^{2}\right)$ can be estimated from the superheating measurement; hence, the performance index (4) can be obtained.

Remark 2. The main advantage of QMEE is that it can decrease the computational complexity compared with MEE. When calculating $I P_{2}\left(e_{k}\right)$ in MEE based on sliding window, whose width is $N$, the computational complexity is $O\left(N^{2}\right)$ due to the existence of inner summation; meanwhile, $I P_{2}^{Q}\left(e_{k}\right)$ can be calculated according to (5) adopting the quantizing error samples, which reduces the number of inner summations. 


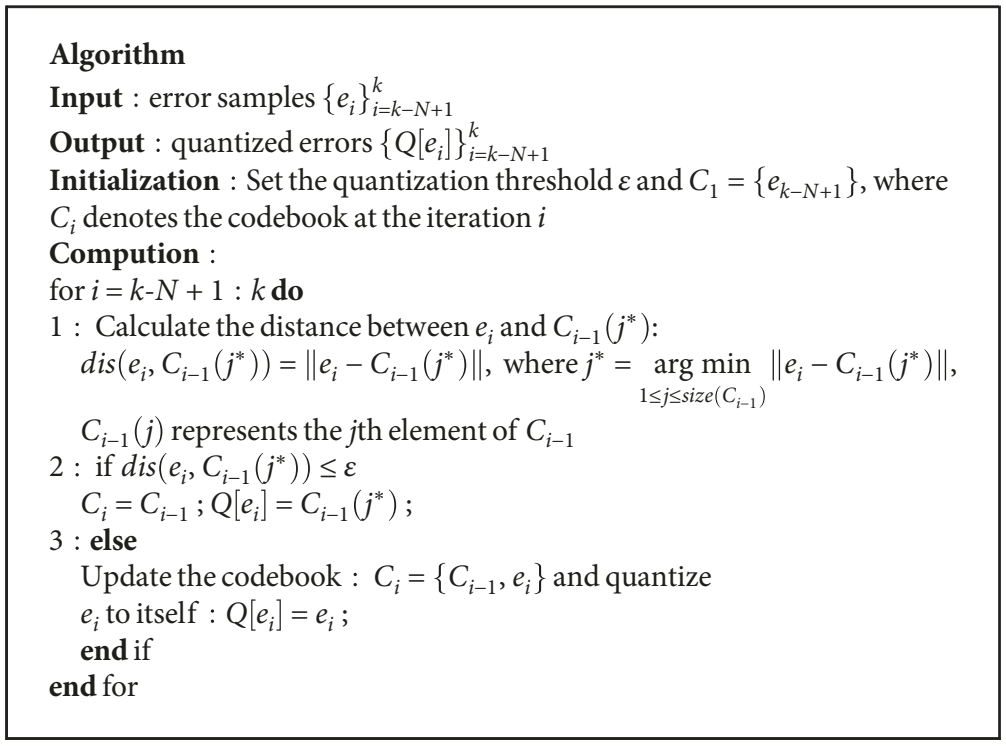

Algorithm 1: Pseudocode of estimating $I P_{2}^{Q}\left(e_{k}\right)$.

And its computational burden is $O(M N)$ with $M \ll N$, especially for large-scale datasets.

Remark 3. Tracking error samples should contain the dynamic characteristics of the ORC process. $N$ error samples $\left\{e_{k}, e_{k-1}, \ldots, e_{k-N+1}\right\}$, which are used to estimate $I P_{2}^{Q}\left(e_{k}\right)$ at instant $k$, can be collected using a sliding window whose width is $N$.

3.3. Optimal Controller via PSO. The optimal control input $u_{k}^{*}$ can be obtained by minimizing the performance index (4) using particle swarm optimization (PSO) algorithm. PSO is a bio-inspired optimal algorithm, which adopts global search strategy based on population and a simple velocity-displacement model. The fundamental notion of PSO can be represented by the following description. Assuming that $G$ is the maximum generation, in each iteration, the particle updates itself by tracking two optimal values: (1) Pbest $^{i(t)}$ symbolizes the individual optimal value, which represents the optimal location of the current particle; (2) another optimal value is the optimal solution Gbest founded in the whole group. Then, the particles pursue the current optimal particles and search in the solution space.

Assuming a $D$ dimension target search space, a group of $L$ particles, and the expressions of the position and velocity of the $i^{\text {th }}$ particle are as follows:

$$
\begin{aligned}
u_{k}^{i(t)} & =\left(u_{k}^{i 1(t)}, u_{k}^{i 2(t)}, u_{k}^{i 3(t)}, \ldots, u_{k}^{i D(t)}\right), \\
V^{i(t)} & =\left(v^{i 1(t)}, v^{i 2(t)}, v^{i 3(t)}, \ldots, v^{i D(t)}\right), \\
i & =1,2,3, \ldots, L,
\end{aligned}
$$

where $t$ is the pointer of iterations. The updated position and velocity of the particle in the $t+1$ generation can then be acquired with the following formulas:

$$
\begin{aligned}
V^{i(t+1)}= & w \cdot V^{i(t)}+c_{1} \cdot \text { rand } \cdot\left(\text { Pbest }^{i(t)}-u_{k}^{i(t)}\right) \\
& +c_{2} \cdot \text { rand } \cdot\left(\text { Gbest }^{t}-u_{k}^{i(t)}\right), \\
u_{k}^{i(t+1)}= & u_{k}^{i(t)}+V^{i(t+1)}, \\
i= & 1,2,3, \ldots, L,
\end{aligned}
$$

where $w$ is the inertia weight factor and satisfies $0 \leq w<1 . c_{1}$ and $c_{2}$ are the acceleration factors.

In order to maintain the global searching ability of the particle in the early stages and the local search ability in the later period, the inertia weight reduction strategy is adopted, that means using a larger inertia weight at the beginnings of the algorithm to search the whole problem space effectively, and using a smaller inertia weight in the later period ensure the convergence of the algorithm. The basic idea can be described by

$$
w=w_{\max }-\frac{w_{\max }-w_{\min }}{G} \cdot t
$$

After $G$ generations, the homologous Gbest can be deemed as the optimal control law at instant $k$; it leads to

$$
u_{k}^{*}=\text { Gbest. }
$$

Instead of the traditional gradient descent method, PSO is used to obtain the optimal control law at instant $k$, in which the performance index (4) is used as the fitness function; the 


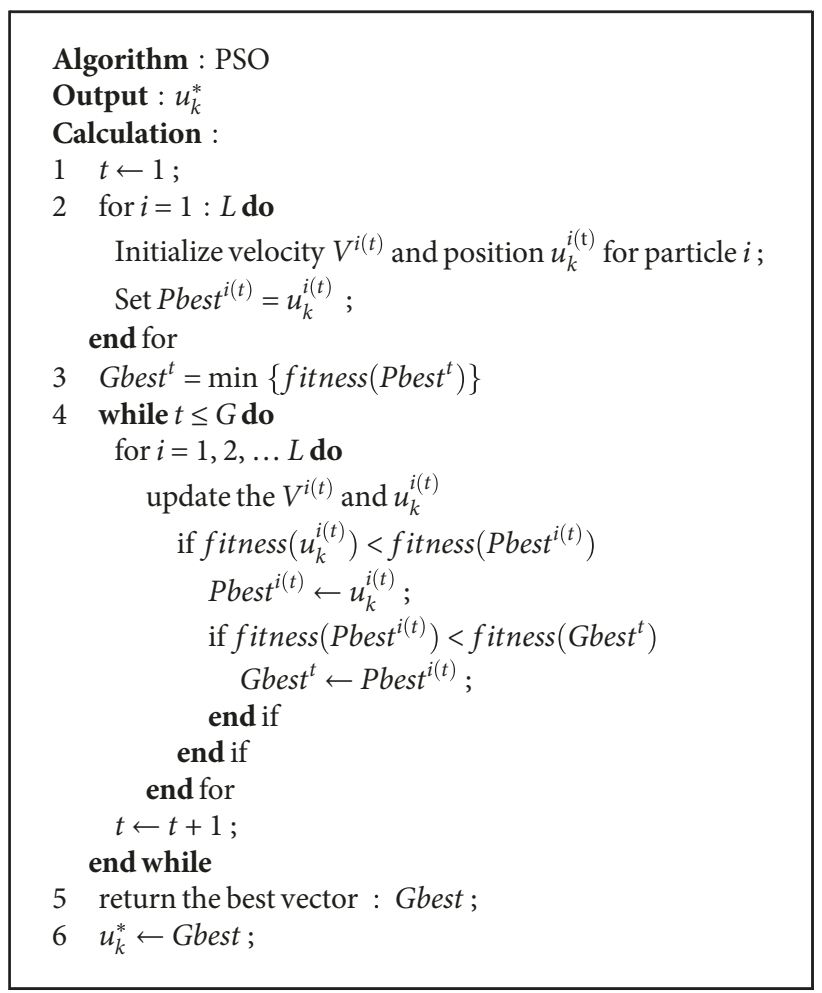

Algorithm 2: Pseudocode of solving optimal control input.

procedures to solve the optimal control input $u_{k}^{*}$ are summarized in Algorithm 2.

Remark 4. The control signal generated by the PSO method constructs an output feedback law for nonlinear and non-Gaussian systems. Following the stability analysis in [32], the stability of the closed-loop system can be analyzed as well. The uniform boundedness of all the variables inside the closed loop can be guaranteed if the absolute value of the incremental control input is bounded, i.e., $\left|\Delta u_{k}\right| \leq U_{\max }-U_{\min }$, where $U_{\max }$ and $U_{\min }$ stand for the maximum and minimum of rotor speed of the pump.

The implementation procedures of the presented superheating control system are summarized as follows:

Step 1. Initialize the parameters of the proposed control algorithm

Step 2. Calculate the performance index (4) by combing the quantized quadratic information potential of tracking error using samples within sliding window, mean value of squared errors, and constraints on control input

Step 3. Solve the optimal control law by minimizing the performance index (4) using the PSO algorithm

Step 4. Apply the optimal control input to regulate the superheating. Then, repeat the procedures from step 2 to step 4 for the next instant, $k=k+1$

\section{Simulation Results}

The proposed control approach is applied to control the superheating of an ORC process [11]. Simulation experiments are carried out with MATLAB 2014b, running in i7-4790, 3.6 GHz CPU. The sampling period is $T=20$ s. In this simulation, the rotating speed of the pump is limited in terms of $2810 \mathrm{rpm} \leq u_{k} \leq 2855 \mathrm{rpm}$. The probability distributions of the mass flow $w_{k}$ and temperature $v_{k}$ of waste heat source are shown in Figure 3. It is clear that the data of $w_{k}$ and $v_{k}$ cannot be fitted exactly to a straight line in the normal probability plot. As a result, it indicates that $w_{k}$ and $v_{k}$ are non-Gaussian. The parameters in the simulation are set as Table 1.

In order to testify the effectiveness of the proposed control method, it is compared with an optimal control method called PSO-MEE and optimal PID controller, respectively. The PSO-MEE-based optimal control law solves optimal control using performance index (3). The proposed control law called PSO-QMEE is obtained by minimizing performance index (4) which contains the quantized entropy of (the tracking) error. The optimal parameters of the PID controller whose transfer function is $G_{\mathrm{PID}}(s)=k_{p}+k_{i} / s+k_{d} s$ are tuned by MATLAB $2014 \mathrm{~b}$ software; it leads to $k_{p}=10.315, k_{i}=8.022$, and $k_{d}=1$.

Figure 4 shows the superheating response curves under three kinds of controller, in which the black dash-dot line shows the set point changes from $17^{\circ} \mathrm{C}$ to $19^{\circ} \mathrm{C}$ at $1500 \mathrm{~s}$. 

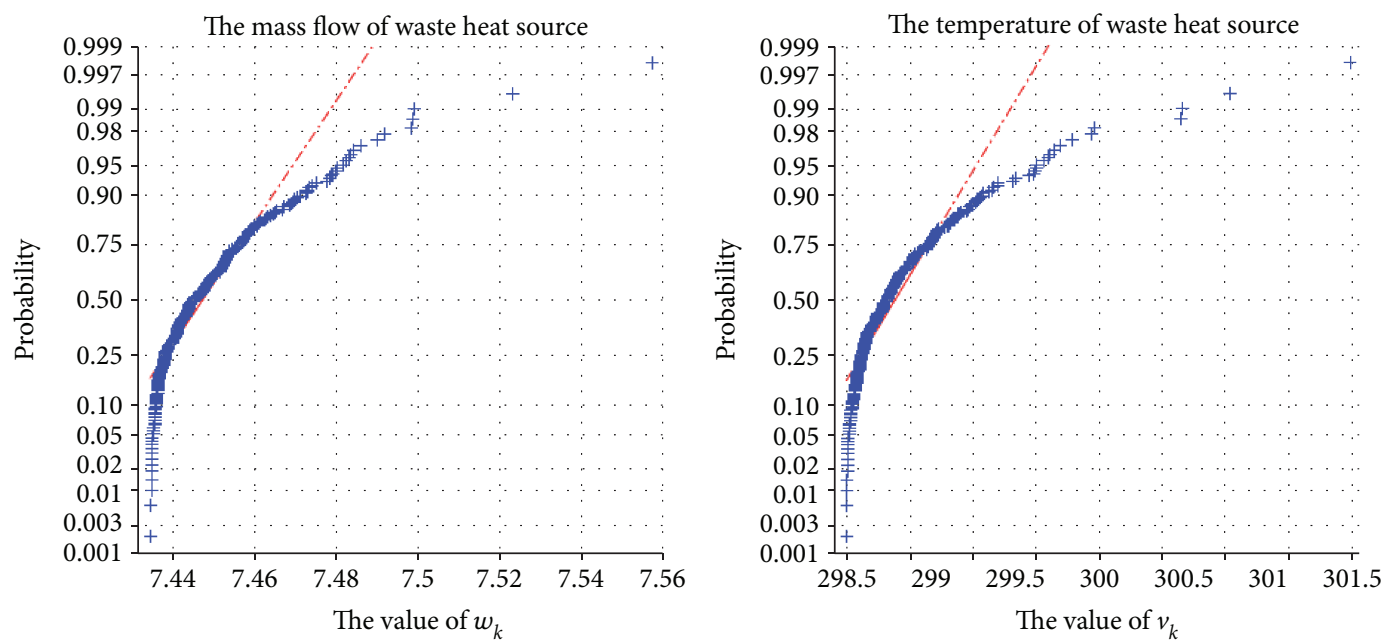

Figure 3: Probability of $w_{k}$ and $v_{k}$.

TABLE 1: Parameters in the simulation.

\begin{tabular}{lccc}
\hline Parameter & Value & Parameter & Value \\
\hline$c_{1}$ & 1.49445 & {$\left[V_{\min }, V_{\max }\right]$} & {$[-5,5]$} \\
$c_{2}$ & 1.49445 & {$\left[w_{\min }, w_{\max }\right]$} & {$[0.1,0.9]$} \\
$\varepsilon$ & 0.1 & $G$ & 50 \\
$R_{1}$ & 0.1 & $L$ & 5 \\
$R_{2}$ & 0.5 & $R_{3}$ & 0.01 \\
\hline
\end{tabular}

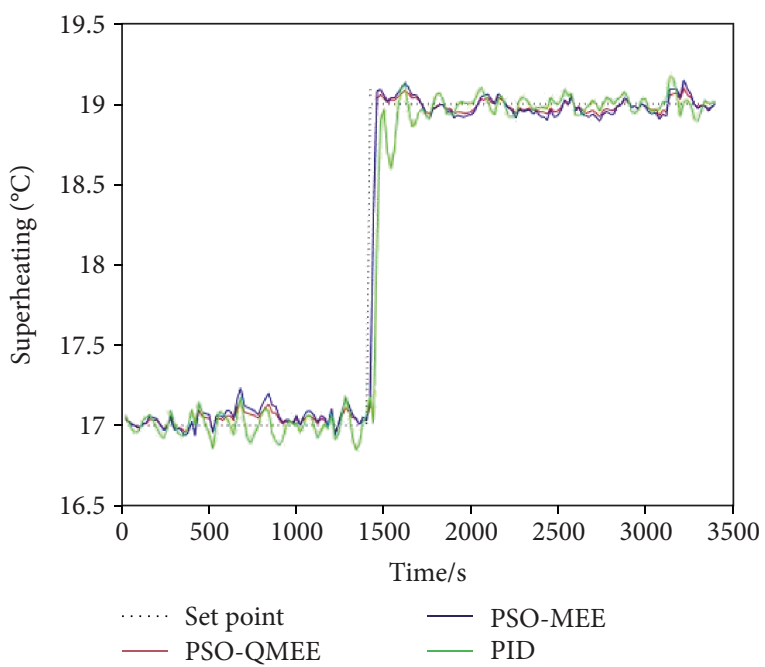

FIGURE 4: Response curves of superheating.

The red solid line, blue solid line, and green solid line represent superheating responses under PSO-QMEE, PSO-MEE, and PID controller, respectively. Compared with the PID controller, both the proposed control algorithm and the conventional entropy-based control obtain smaller overshoot and shorter settling time. Table 2 lists detailed comparative results when using three controllers.
TABLE 2: Comparative results using three controllers.

\begin{tabular}{lccc}
\hline Controllers & MSE & $\begin{array}{c}\text { Times for computing } \\
\text { control laws in overall } \\
\text { simulation process }\end{array}$ & MAE \\
\hline Proposed control & 0.0258 & $82.77661 \mathrm{~s}$ & 0.0526 \\
PID & 0.0527 & 0 & 0.0826 \\
$\begin{array}{l}\text { Conventional } \\
\text { entropy-based control }\end{array}$ & 0.0271 & $1213.08278 \mathrm{~s}$ & 0.0709 \\
\hline
\end{tabular}

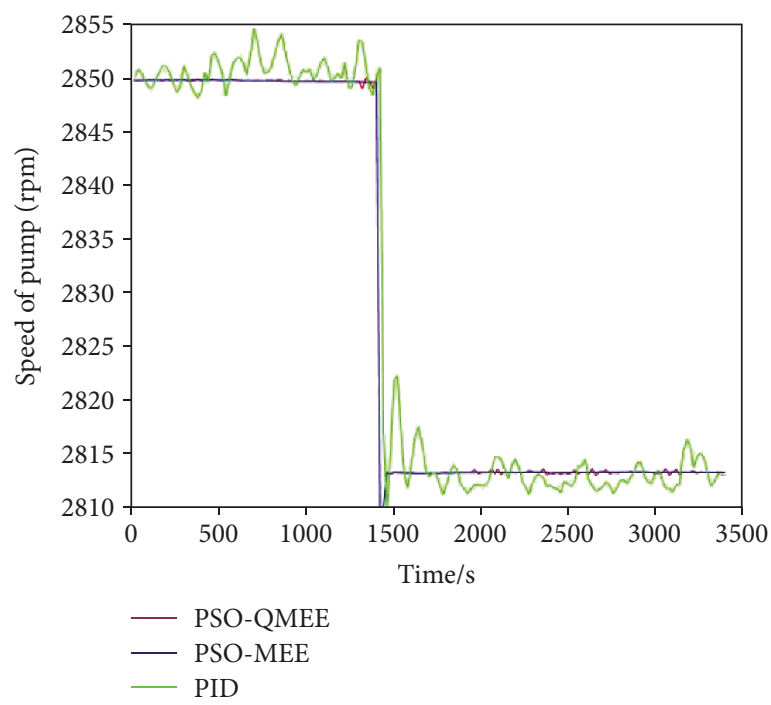

FIgURE 5: Variations of the rotating speed of the pump.

The proposed controller achieves the smallest mean square error (MSE) and mean absolute error (MAE); moreover, it consumes shorter time than the conventional entropy-based controller due to using quantized entropy estimation method. Figure 5 demonstrates variations of the rotating speed of the pump under three control laws; 
it can be observed that the manipulated signals are all reasonable and feasible.

\section{Conclusions}

In this paper, a data-driven superheating controller is presented to regulate the superheating in ORC processes. Since the disturbances coming from the mass flow rate and the temperature of waste heat are not necessarily Gaussian, the performance index is constructed by combing the entropy of the tracking error, the mean value of the squared tracking error, and constraints on control input. The entropy of the tracking error is replaced by quantized information potential of the tracking error for decreasing computational burden. Consequently, the superheating control problem is solved using the PSO method. When applying the proposed controller, conventional entropy-based controller, and a PID controller to the ORC system, respectively, the proposed controller can decrease dispersion of the tracking error distribution and computational burden.

\section{Data Availability}

These data used in this research have been listed in Table 1, Table 2, or described in Section 4. The simulation results in Figures 4 and 5 should be made easily available if other scientists are interested in reanalyzing the data.

\section{Conflicts of Interest}

The authors declare that they have no conflicts of interest.

\section{Acknowledgments}

This work was supported by the Chinese National Science Foundation under Grant (61503271, 61603136, 61374052) and the Fundamental Research Funds for the Central Universities (2016ZZD03). These are gratefully acknowledged.

\section{References}

[1] M. Wei, P. Song, B. Zhao, L. Shi, Z. Wang, and C. Ma, "Unsteady flow in the suction process of a scroll expander for an ORC waste heat recovery system," Applied Thermal Engineering, vol. 78, pp. 460-470, 2015.

[2] J. Xu and C. Yu, "Critical temperature criterion for selection of working fluids for subcritical pressure organic Rankine cycles," Energy, vol. 74, no. 5, pp. 719-733, 2014.

[3] G.-D. Xia, Y.-Q. Zhang, Y.-T. Wu et al., "Experimental study on the performance of single-screw expander with different inlet vapor dryness," Applied Thermal Engineering, vol. 87, pp. 34-40, 2015.

[4] F. Yang, X. Dong, H. Zhang et al., "Performance analysis of waste heat recovery with a dual loop organic Rankine cycle (ORC) system for diesel engine under various operating conditions," Energy Conversion and Management, vol. 80, no. 2, pp. 243-255, 2014.

[5] J. Zhang, K. Li, and J. Xu, "Recent developments of control strategies for organic Rankine cycle (ORC) systems," Transactions of the Institute of Measurement and Control, vol. 47, 2018.
[6] S. Quoilin, R. Aumann, A. Grill, A. Schuster, V. Lemort, and H. Spliethoff, "Dynamic modeling and optimal control strategy of waste heat recovery organic Rankine cycles," Applied Energy, vol. 88, no. 6, pp. 2183-2190, 2011.

[7] M. Usman, M. Imran, D. H. Lee, and B.-S. Park, "Experimental investigation of off-grid organic Rankine cycle control system adapting sliding pressure strategy under proportional integral with feed-forward and compensator," Applied Thermal Engineering, vol. 110, pp. 1153-1163, 2017.

[8] A. Torregrosa, J. Galindo, V. Dolz, L. Royo-Pascual, R. Haller, and J. Melis, "Dynamic tests and adaptive control of a bottoming organic Rankine cycle of IC engine using swash-plate expander," Energy Conversion and Management, vol. 126, pp. 168-176, 2016.

[9] J. Peralez, M. Nadri, P. Dufour, P. Tona, and A. Sciarretta, "Organic Rankine cycle for vehicles: control design and experimental results," IEEE Transactions on Control Systems Technology, vol. 25, no. 3, pp. 952-965, 2017.

[10] J. Zhang, W. Zhang, G. Hou, and F. Fang, "Dynamic modeling and multivariable control of organic Rankine cycles in waste heat utilizing processes," Computers \& Mathematics with Applications, vol. 64, no. 5, pp. 908-921, 2012.

[11] J. Zhang, Y. Zhou, R. Wang, J. Xu, and F. Fang, "Modeling and constrained multivariable predictive control for ORC (organic Rankine cycle) based waste heat energy conversion systems," Energy, vol. 66, pp. 128-138, 2014.

[12] G. Hou, S. Bi, M. Lin, J. Zhang, and J. Xu, "Minimum variance control of organic Rankine cycle based waste heat recovery," Energy Conversion and Management, vol. 86, pp. 576-586, 2014.

[13] R. Shi, T. He, J. Peng, Y. Zhang, and W. Zhuge, "System design and control for waste heat recovery of automotive engines based on organic Rankine cycle," Energy, vol. 102, pp. 276286, 2016.

[14] J. Zhang, M. Lin, F. Fang, J. Xu, and K. Li, "Gain scheduling control of waste heat energy conversion systems based on an LPV (linear parameter varying) model," Energy, vol. 107, pp. 773-783, 2016.

[15] J. Zhang, M. Lin, J. Chen, J. Xu, and K. Li, "PLS-based multiloop robust $\mathrm{H} 2$ control for improvement of operating efficiency of waste heat energy conversion systems with organic Rankine cycle," Energy, vol. 123, pp. 460-472, 2017.

[16] H. Wang, Bounded Dynamic Stochastic Systems: Modelling and Control, Springer, 2000.

[17] H. Wang and J. Hua Zhang, "Bounded stochastic distributions control for pseudo-armax stochastic systems," IEEE Transactions on Automatic Control, vol. 46, no. 3, pp. 486-490, 2001.

[18] H. Wang and P. Afshar, "ILC-based fixed-structure controller design for output PDF shaping in stochastic systems using LMI techniques," IEEE Transactions on Automatic Control, vol. 54, no. 4, pp. 760-773, 2009.

[19] L. Guo and H. Wang, "Stochastic distribution control system design," in Advances in Industrial Control, Springer, 2010.

[20] P. Afshar, H. Wang, and T. Chai, “An ILC-based adaptive control for general stochastic systems with strictly decreasing entropy," IEEE Transactions on Neural Networks, vol. 20, no. 3, pp. 471-482, 2009.

[21] Y. Liu, H. Wang, and L. Guo, "Observer-based feedback controller design for a class of stochastic systems with nonGaussian variables," IEEE Transactions on Automatic Control, vol. 60, no. 5, pp. 1445-1450, 2015. 
[22] Y. Zhou, Q. Zhang, H. Wang, P. Zhou, and T. Chai, "EKFbased enhanced performance controller design for non-linear stochastic systems," IEEE Transactions on Automatic Control, vol. 63, no. 4, pp. 1155-1162, 2018.

[23] Q. Zhang and X. Yin, "Observer-based parametric decoupling controller design for a class of multi-variable non-linear uncertain systems," Systems Science \& Control Engineering, vol. 6, no. 1, pp. 258-267, 2018.

[24] S. Peng, W. Ser, B. Chen, L. Sun, and Z. Lin, "Robust constrained adaptive filtering under minimum error entropy criterion," IEEE Transactions on Circuits and Systems II: Express Briefs, vol. 65, no. 8, pp. 1119-1123, 2018.

[25] B. Chen, X. Wang, N. Lu, S. Wang, J. Cao, and J. Qin, "Mixture correntropy for robust learning," Pattern Recognition, vol. 79, pp. 318-327, 2018.

[26] B. Chen, J. Hu, L. Pu, and Z. Sun, "Stochastic gradient algorithm under (h, $\varphi$ )-entropy criterion," Circuits, Systems \& Signal Processing, vol. 26, no. 6, pp. 941-960, 2007.

[27] M. Ren, J. Zhang, M. Jiang, M. Yu, and J. Xu, "Minimum($h, \phi)$-entropy control for non-Gaussian stochastic networked control systems and its application to a networked DC motor control system," IEEE Transactions on Control Systems Technology, vol. 23, no. 1, pp. 406-411, 2015.

[28] J. C. Principe, Information Theoretic Learning: Renyi's Entropy and Kernel Perspectives, Springer, 2010.

[29] B. Chen, L. Xing, N. Zheng, and J. C. Príncipe, "Quantized minimum error entropy criterion," IEEE Trans. on Neural Networks and Learning Systems, 2017, https://arxiv.org/abs/ 1710.04089 .

[30] B. Chen, S. Zhao, P. Zhu, and J. C. Principe, "Quantized kernel least mean square algorithm," IEEE Transactions on Neural Networks and Learning Systems, vol. 23, no. 1, pp. 22-32, 2012.

[31] J. Zhang, C.-C. Chu, J. Munoz, and J. Chen, "Minimum entropy based run-to-run control for semiconductor processes with uncertain metrology delay," Journal of Process Control, vol. 19, no. 10, pp. 1688-1697, 2009.

[32] H. Yue and H. Wang, "Minimum entropy control of closedloop tracking errors for dynamic stochastic systems," IEEE Transactions on Automatic Control, vol. 48, no. 1, pp. 118122, 2003. 


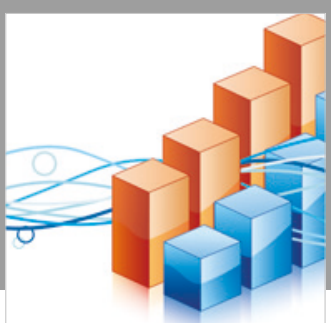

Advances in

Operations Research

\section{-n-m}
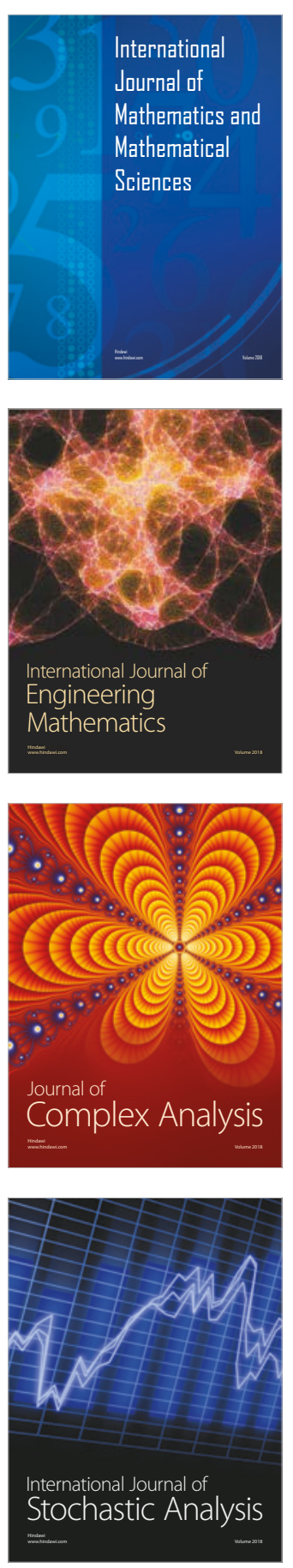
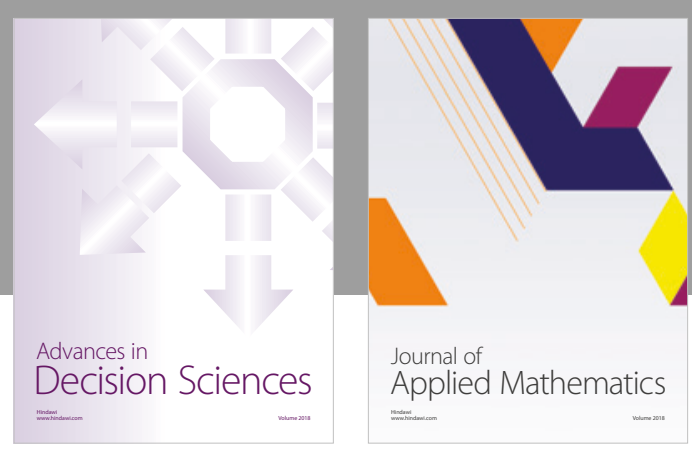

Journal of

Applied Mathematics
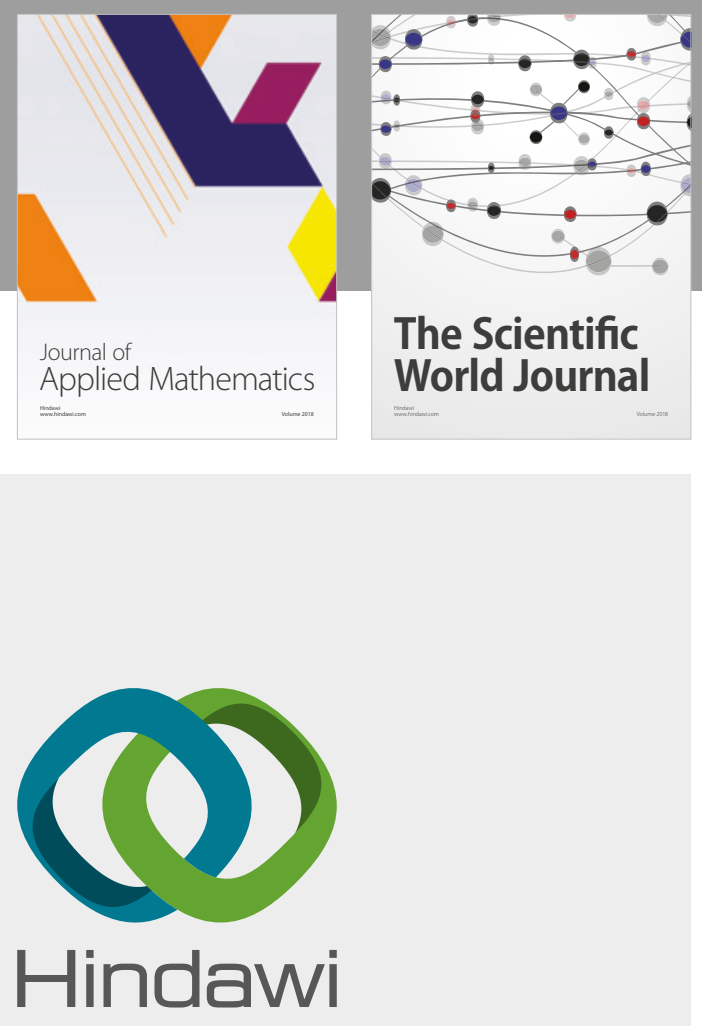

Submit your manuscripts at

www.hindawi.com

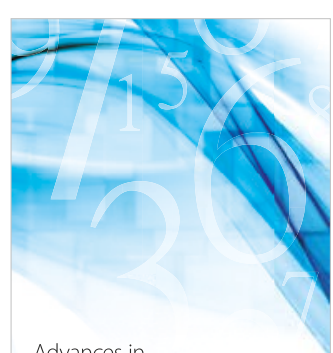

Advances in
Numerical Analysis
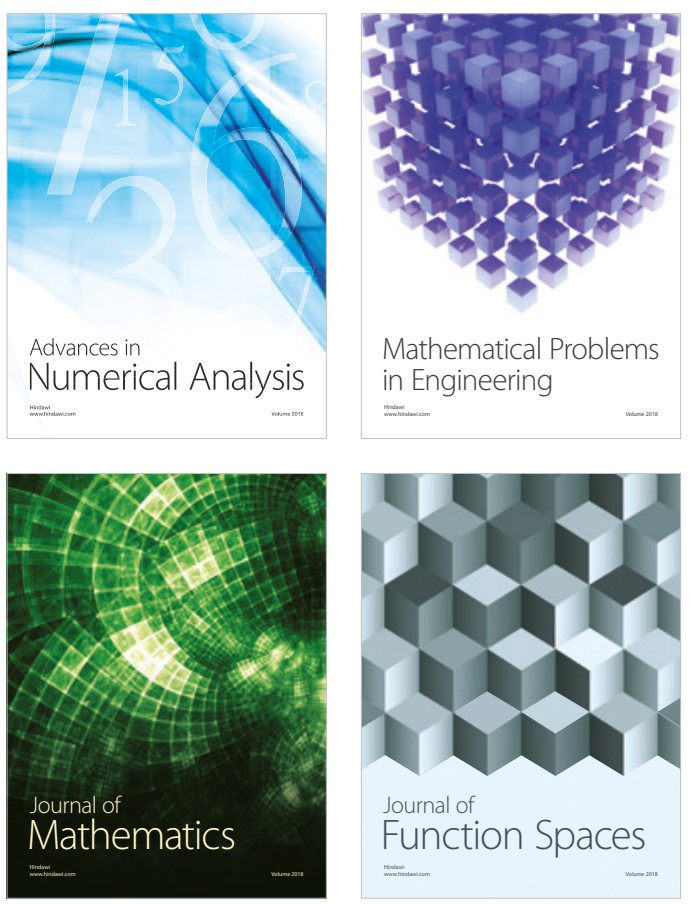

Mathematical Problems in Engineering

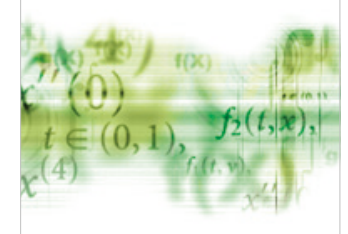

International Journal of

Differential Equations

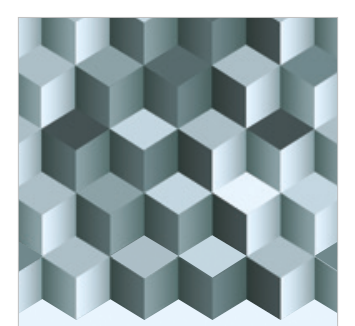

Journal of

Function Spaces
The Scientific

World Journal

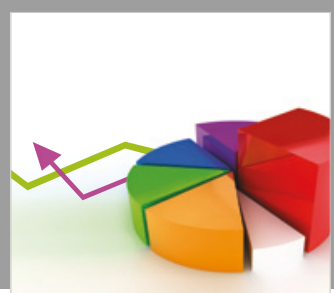

Journal of

Probability and Statistics
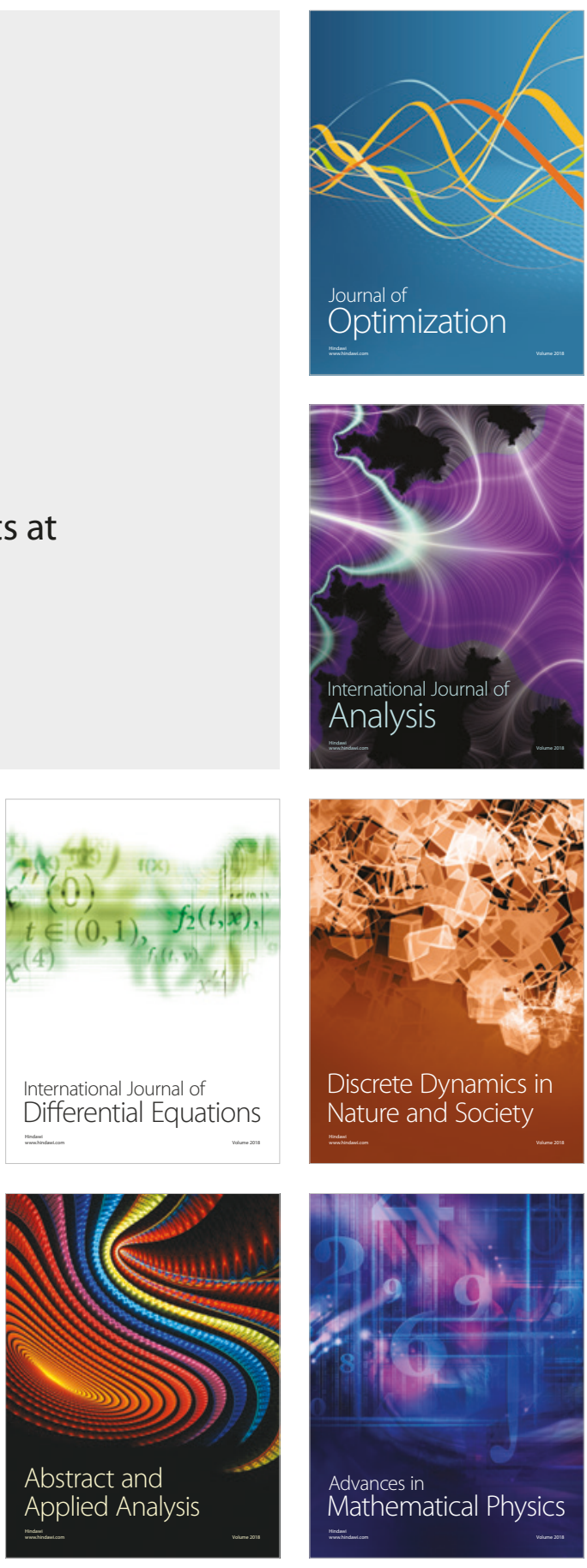